\title{
An audit of ANCA in routine clinical practice
}

\author{
JDM Edgar, SA McMillan, IN Bruce, SK Conlan
}

\begin{abstract}
Summary
We have reviewed the medical records of $301 / 327$ consecutive patients in whom anti-neutrophil cytoplasmic antibodies (ANCA) were detected by the Regional Immunology Laboratory in Northern Ireland between January 1988 and October 1991 (45 months). We have collected data for each patient regarding age, sex, smoking habit, area of residence, and details of any other autoantibody activity. Clinical diagnosis was established, with the number of organ systems involved and the evidence for that involvement (symptomatic, biochemical, radiological, and histological). Diagnoses were divided into four groups according to their recognised vasculitic features and these were related to the pattern of immunofluorescence and maximum ANCA titre detected.

The most frequent diagnosis was rheumatoid arthritis (18.2\% of patients) and the connective tissue disorders as a whole accounted for $27.9 \%$ of patients. ANCA were also detected in a wide range of clinical conditions which are not associated with vasculitis and these patients were an important source of 'false-positives'. The positive predictive value (PPV) of ANCA of all patterns and titres for vasculitic conditions was $27 \%$, however, the detection of a classical ANCA pattern at high titre $(\geqslant 1: 640)$ was associated with an increased PPV of $75 \%$. The coexistence of an antinuclear antibody (ANA) reduces the PPV of both classical and perinuclear ANCA, although perinuclear ANCA with antimyeloperoxidase specificity had an improved PPV.

We conclude that ANCA testing should not be used as the only screening investigation for vasculitis but should be included in a rational investigative scheme. The interpretation of a positive ANCA result must take into account the presence of other autoantibodies and the full range of non-vasculitic conditions when the clinical situation is not typical of vasculitis.
\end{abstract}

Keywords: ANCA, clinical interpretation, audit

Rheumatology, Royal

Victoria and Musgrave

Park Hospitals,

Belfast, N Ireland

IN Bruce

Accepted 19 April 1995
Anti-neutrophil cytoplasmic antibodies (ANCA) were first described in 1982 by Davies et $a l^{1}$ in eight patients with segmental necrotising glomerulonephritis. Subsequent studies indicated that ANCA were both highly sensitive and specific markers for Wegener's granulomatosis, ${ }^{2}$ and also surrogate markers of disease activity. ${ }^{3}$ The introduction of ANCA into diagnostic service has therefore contributed greatly to the rapid recognition and management of patients with potentially lifethreatening conditions. Since their original description, however, it has become clear that ANCA occur in a spectrum of vasculitic conditions in addition to Wegener's granulomatosis. $^{4,5}$ ANCA have also been reported in a diversity of other conditions including acute infection, inflammatory bowel disease, rheumatoid arthritis and neoplasia.$^{6-11}$

ANCA are detected by indirect immunofluorescence using alcohol-fixed human neutrophils as substrate. ${ }^{12}$ There are two main staining patterns: classical (cANCA) and perinuclear (pANCA), each associated with different subcellular antigenic specificity. The majority of cANCA are specific for proteinase 3, whilst the best characterised pANCA antigen is myeloperoxidase. Additional specificities include CAP 57 (cANCA) and lactoferrin, elastase, lysozyme and cathepsin $G$ (pANCA). The different patterns on indirect immunofluorescence and antigenic specificities have been correlated with particular disease manifestations ie: cANCA with Wegener's granulomatosis and pANCA with renal limited disease. Intermediate staining patterns have more recently been reported: XANCA in ulcerative colitis ${ }^{9,10}$ and atypical aANCA are reported to be associated with lactoferrin specificity in Felty's syndrome. ${ }^{11}$ In vivo studies have demonstrated that ANCA titre correlates with disease activity ${ }^{3}$ and ANCA have been implicated in neutrophil-mediated damage to endothelial cells in vitro. ${ }^{13}$ This suggests but does not prove, a primary pathogenetic role for these autoantibodies in the causation of vasculitis, and a model for their role has been suggested. ${ }^{4,14,15}$

The increasing diversity of clinical associations of ANCA has made the clinical interpretation of a positive ANCA result more difficult and we therefore felt it was timely to review the disease associations of ANCA detected in routine clinical practice. Our aim was to define more clearly the predictive value of an ANCA result for a defined group of primary vasculitic conditions and establish the relative frequency of other conditions causing 'false-positive' results. 


\section{Patients and methods}

From laboratory records we determined that during the period from 1 January 1988 to 1 October 1991, 327 patients from the local community had ANCA detected. Medical records of all these patients were requested for review and a database was established (SPSS/ $\mathrm{PC}+$, Microsoft Corp, 1988) to include information for each patient. Information was recorded regarding patients age, sex, smoking habit and geographical area of residence (by postcode). The date and titre of ANCA detected was recorded; serially for patients in whom multiple samples were received.

The primary diagnosis was accepted as that which the consultant physician in charge had established in the light of all available clinical and pathological information. Where doubt existed regarding diagnosis following chart review, the physician was directly contacted for advice. The ANCA requests mainly involved patients undergoing secondary or tertiary referral to specialists; $66(21.9 \%)$ were from renal physicians, $60(19.9 \%)$ from rheumatologists, $21(7 \%)$ from gastroenterologists, 18 $(6 \%)$ from respiratory physicians, and 107 $(35.5 \%)$ from general physicians. It is important to recognise, however, that despite rigorous review of diagnosis, it is possible that a small percentage of patients may have had unrecognised occult vasculitic disease. Similarly it is impossible to completely disregard the influence of a positive ANCA result on the diagnostic process.

Vasculitis may be classified in many different ways, classifications being based on vessel size, presence or absence of granulomata, or on the perception of whether the vasculitis process is primary or secondary. For the purposes of this study we divided patients into four groups (see table 1). Group 1 consisted of the recognised vasculitides, ${ }^{16}$ Group 2 consisted of the connec-

Table 1 Group $1-3$ patients $(n=183)$

\begin{tabular}{lccc}
\hline Diagnosis & $\begin{array}{c}p A N C A \\
(n=112)\end{array}$ & $\begin{array}{l}c A N C A \\
(n=71)\end{array}$ & $\begin{array}{l}\text { Total } \\
(n=183)\end{array}$ \\
\hline Group 1 & & & \\
Wegener's granulomatosis & 5 & 14 & 19 \\
Microscopic polyangitis & 19 & 13 & 32 \\
Polyarteritis & 2 & 3 & 5 \\
Syndrome of systemic vasculitis & 6 & 9 & 15 \\
Henoch-Schonlein purpura & 1 & 1 & 2 \\
Behcet's syndrome & 1 & 0 & 1 \\
Leucocytoclastic vasculitis & 1 & 1 & 2 \\
Churg-Strauss syndrome & 1 & 4 & 5 \\
Group 2 & & & \\
Rheumatoid arthritis & 40 & 15 & 55 \\
Systemic lupus erythematosus & 21 & 1 & 22 \\
Scleroderma & 2 & 0 & 2 \\
Mixed connective tissue disease & 2 & 0 & 2 \\
Undifferentiated connective tissue disorder & 1 & 1 & 2 \\
Juvenile chronic arthritis & 1 & 0 & 1 \\
Group 3 & & & \\
Ulcerative colitis & 0 & 4 & 4 \\
Crohn's disease & 5 & 4 & 9 \\
Sclerosing cholangitis & 2 & 0 & 2 \\
Polymyalgia rheumatica & 2 & 1 & 3 \\
\hline & & & \\
\hline
\end{tabular}

tive tissue disorders, this group of conditions having variable secondary vasculitic conditions. Group 3 included conditions not typically associated with vasculitic clinical features. Finally, Group 4 included patients with non-vasculitic conditions and patients with no final diagnosis. Whilst several of the 'diagnoses' cited in this group are simply clinically descriptive, eg, chronic renal failure, spastic paraparesis and microscopic haematuria they are included for completeness.

The number of organ systems recognised to be affected by the disease process was recorded and the nature of this involvement was noted (symptomatic, biochemical, radiological, other). Where histological confirmation of diagnosis had been sought this was noted with the nature of biopsy and whether this had been supportive, non-supportive or non-specific regarding the clinical diagnosis. The use of immunosuppressive therapy and secondary diagnosis was also recorded where appropriate. All other autoantibody activity detected was detailed including specificity, class and titre.

ANCA were detected by the standard indirect immunofluorescent technique using an IgG-specific conjugate. ${ }^{12}$ Sera were screened at a dilution of $1: 10$ and final titre was determined using doubling serum dilutions. Our laboratory is a member of the UK external quality assurance scheme for ANCA. One of us (SKC) read all the ANCA immunofluorescence to exclude potential inter-observer variability. Positive ANCA results were described as either c or pANCA. All positive sera were further tested using an anti-myeloperoxidase (antiMPO) enzyme-linked immunosorbent assay (ELISA, Biocarb Diagnostics AB Sweden). An anti-MPO value of greater than 20 ELISA Units (EU) was regarded as positive, based on the upper 95 th percentile of a normal population ( $n=50$, unpublished data). Autoantibody testing was performed by indirect immunofluorescence using a composite multiblock substrate (mouse stomach, rat liver and kidney: Biodiagnostics Ltd, UK) on each sample and antinuclear antibodies were confirmed and further characterised using a Hep2 cell line substrate (Biodiagnostics Ltd, UK).

\section{Results}

PATIENTS

We reviewed the records of 301 patients which represents $92 \%$ of all local patients in whom ANCA was detected during the study period. Characteristics of the sample population are shown in box 1. Other than a slight preponderance of smokers in the cANCA positive subset $(38.1 \%$ vs $26.8 \%)$ there was no significant difference in these characteristics between the pANCA and cANCA positive subgroups. There was no obvious geographical clustering of the patients reviewed.

\section{DIAGNOSIS AND ANCA DETECTION}

Diagnoses are listed (in tables 1 and 2) with the total numbers and relative frequencies of cANCA and pANCA detection. Of the 301 


\begin{tabular}{|ll|}
\hline $\begin{array}{l}\text { Characteristics of patient popula- } \\
\text { tion }(\boldsymbol{n}=301)\end{array}$ \\
\hline $\begin{array}{ll}\text { - p/c ANCA } \\
\text { - male/female }\end{array}$ & $183 / 118$ \\
- smokers/non-smokers & $135 / 166$ \\
- mean age (years) & $94 / 171$ \\
\hline
\end{tabular}

Box 1

patients, only $81(27 \%)$ had conditions from Group 1. Biopsy was performed in $60 \%$ of these patients and was supportive of the clinical diagnosis in 41 cases $(51 \%$ ).

A total of 84 patients had one of the connective tissue disorders, rheumatoid arthritis being the most frequent diagnosis in both $\mathrm{c}$ and pANCA subgroups (table 1). In addition to their characteristic erosive joint disease the ANCA-positive patients with rheumatoid arthritis were characterised as rheumatoid factor positive $(40 / 55)$ with a higher than expected frequency of extra-articular disease (17 had joint disease only; 38 had extra-articular manifestations). The most frequent extra-

Table 2 Group 4 patients $(n=118)$

\begin{tabular}{llll}
\hline Diagnosis & $p$ ANCA & cANCA & Total \\
& $(n=71)$ & $(n=47)$ & $(n=118)$ \\
\hline Chronic renal failure & 7 & 10 & 17 \\
Malignancy & 5 & 8 & 13 \\
Infection & 4 & 6 & 10 \\
Ischaemic heart disease & 6 & 1 & 7 \\
Acute renal failure & 4 & 2 & 6 \\
Chronic active hepatitis & 4 & 0 & 4 \\
Chronic obstructive airways disease & 1 & 2 & 3 \\
Deep venous thrombosis & 3 & 0 & 3 \\
Cryptogenic fibrosing alveolitis & 3 & 0 & 3 \\
Valvular heart disease & 2 & 0 & 2 \\
Microscopic haematuria & 0 & 2 & 2 \\
Osteoarthritis & 0 & 2 & 2 \\
Peripheral neuropathy & 1 & 0 & 1 \\
Spastic paraparesis & 1 & 0 & 1 \\
Sarcoid & 1 & 0 & 1 \\
Polymyositis & 1 & 0 & 1 \\
Cholecystitis & 1 & 0 & 1 \\
Alcohol-induced rhabdomyolysis & 1 & 0 & 1 \\
Emphysema & 0 & 1 & 1 \\
Monoconal gammopathy of uncertain & 1 & 0 & 1 \\
$\quad$ significance & & & \\
Amyloid & 0 & 1 & 1 \\
Retroperitoneal fibrosis & 25 & 11 & 36 \\
No diagnosis & & 1 & \\
\hline & & & 1 \\
\hline
\end{tabular}

Table 3 Extent of organ involvement and biopsy support for diagnosis

\begin{tabular}{lccccc}
\hline & $\begin{array}{c}\text { Group 1 } \\
(n=81)\end{array}$ & $\begin{array}{l}\text { Group 2 } \\
(n=84)\end{array}$ & $\begin{array}{l}\text { Group 3 } \\
(n=18)\end{array}$ & $\begin{array}{l}\text { Group 4 } \\
(n=118)\end{array}$ & $\begin{array}{l}\text { Total } \\
(n=301)\end{array}$ \\
\hline $\begin{array}{l}\text { Organs involved } \\
1\end{array}$ & $20(25 \%)$ & $21(25 \%)$ & $14(78 \%)$ & $76(64 \%)$ & $131(43.5)$ \\
2 & $40(49 \%)$ & $43(50 \%)$ & $4(22 \%)$ & $29(25 \%)$ & $116(38.5)$ \\
3 & $16(20 \%)$ & $15(18 \%)$ & 0 & $6(5 \%)$ & $37(13.3)$ \\
4 & $4(5 \%)$ & $4(5 \%)$ & 0 & $1(1 \%)$ & $9(3.0)$ \\
$\begin{array}{l}\text { Biopsy } \\
\text { performed } \\
\text { supportive }\end{array}$ & $49(60 \%)$ & $14(17 \%)$ & $10(56 \%)$ & $44(37 \%)$ & $117(38.9)$ \\
\hline
\end{tabular}

articular involvement was in the kidney (8), skin (8), lung (7), cardiovascular system (6), eyes (3) and central nervous system (CNS) (3). Most (33/55) required at least one immunosuppressive agent (prednisolone, azathioprine, methotrexate or cyclophosphamide). The 22 patients with systemic lupus erythematosus did not have any obvious distinguishing characteristics, 18 had multiple organ involvement and 11 required single/combination drug immunosuppressive therapy. ANA was positive in 21 cases and antibodies to dsDNA in 17. Four of the five patients who were dsDNA antibody negative had biopsy evidence of lupus. These 22 patients had organ involvement as follows: joints (13), kidney (9), skin (7), cardiovascular (5), lung (2) and CNS (2).

There were 18 patients with diagnoses from Group 3. Ten of the 13 patients with inflammatory bowel disease (ulcerative colitis or Crohn's disease) had no apparent extraintestinal involvement although $8 / 13$ required at least oral prednisolone to control their condition.

There were 118 patients in Group 4 (table 2), $36(30 \%)$ having no final diagnosis. The three most frequent diagnoses in this group, ie, chronic renal failure, infection, and malignancy, accounted for $40(49 \%)$ of those who had a definitive diagnosis made. The 13 patients with malignant disease had no evidence of vasculitis and consisted of: transitional cell carcinoma of the bladder (three), one each of chronic myeloid leukaemia, multiple myeloma, non-Hodgkin's lymphoma, adenocarcinoma of the colon, and ovarian carcinoma, and five cases of advanced metastatic disease with no identified primary tumour.

The 10 patients with infection consisted of: bronchopneumonia (three), urinary tract infection (three, one with underlying medullary sponge kidney), and one each with bronchiectasis, pulmonary tuberculosis (presumed), paracolic abscess, and infective endocarditis, (Streptococcus sanguis isolated).

\section{EXTENT OF DISEASE AND HISTOLOGICAL \\ SUPPORT FOR DIAGNOSIS}

Extent of disease was assessed by the number of organ systems involved in the disease process. Table 3 summarises these data for each of the diagnostic groups. Patients in the vasculitic and the connective tissue disorder categories (Groups 1 and 2) had multiple organ involvement in $73 \%$ of cases whereas this figure is reduced to $22 \%$ and $33 \%$ in Groups 3 and 4, respectively. The evidence for secondary organ involvement was based on more than one of the stated criteria (symptomatic, radiological, biochemical, histological) in approximately $50 \%$ of patients in each subgroup.

The biopsy rate for each subgroup is recorded in table 3. Biopsies were taken from: kidney (37), lung (13), bowel (12), skin (11), nasal mucosa (10), liver (6), muscle (3) and others (17). Autopsy was performed in eight cases. Histological examination of biopsy tissue supported the clinical diagnosis in $84\left(70.6^{\circ}{ }_{0}\right)$, was non-specific in $22\left(18.5^{\circ} 0\right)$ and nonsupportive in $\operatorname{six}\left(5^{\circ}{ }_{0}\right)$ cases. 
MAXIMUM ANCA TITRE

The maximum titre of ANCA detected for each patient ranged from 1:10 to 1:5120 (figure 1) with a marked overall skewing in favour of low-titre ANCA. There was no demonstrable relationship between patient's age and titre of ANCA detected. The mean (SD) age of patients with ANCA $\leqslant 1: 80$ was 59.8 (15.1) years, for those with ANCA > 1:80 it was 57.8 (17.5 years). When the titre data are further examined (figure 2), it is clear that titres tend to be higher in the vasculitis-related group compared to Group 4, the non-vasculitic/no diagnosis group. A clinically important exception are the 20 patients in whom ANCA was present at a titre $\geqslant 1: 80$; ANA was negative but

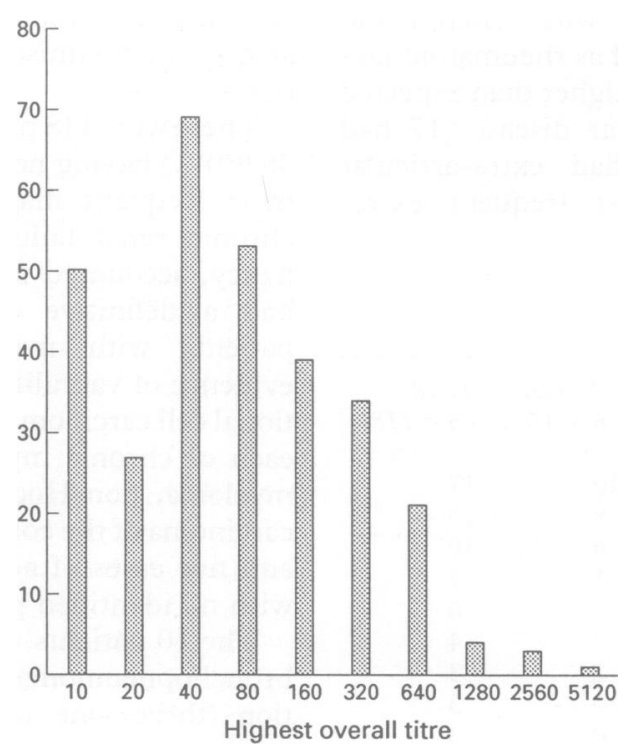

Figure 1 Maximum titre of ANCA detected $(n=301)$
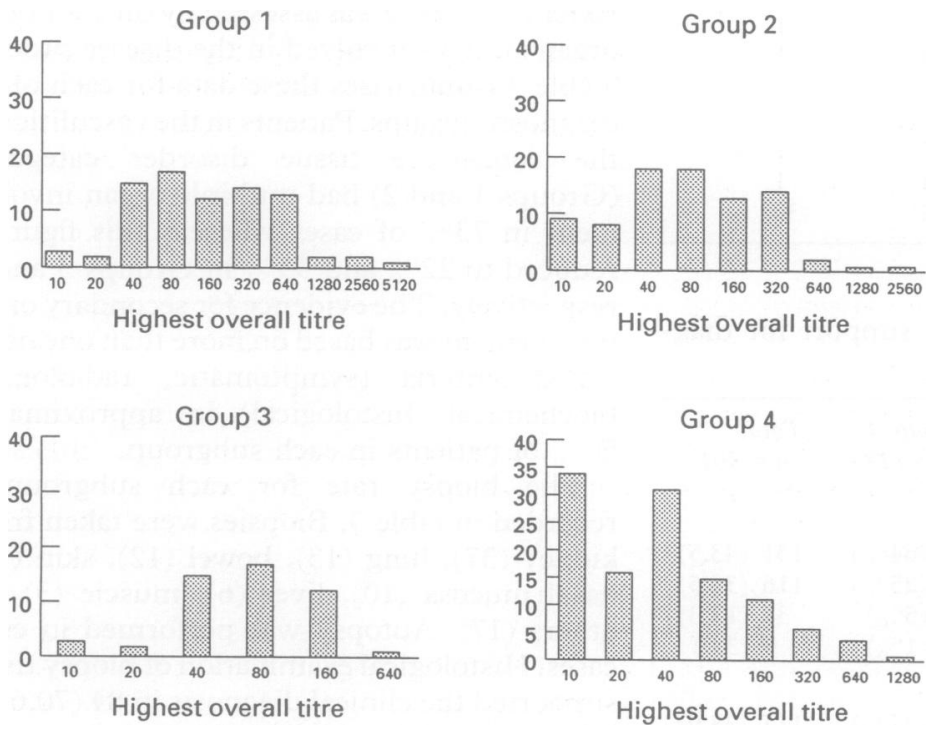

Figure 2 Maximum titre of ANCA detected in each diagnostic group diagnosis was not of vasculitis. Their diagnoses comprised four patients with rheumatoid arthritis, three with Crohn's disease, two with chronic renal failure and one each with polymyalgia rheumatica, scleroderma, chronic obstructive airways disease, neoplasm, undifferentiated connective tissue disorder, and retroperitoneal fibrosis; $75 \%$ of this group were aged 50 or above and eight had titres of $1: 640$ or greater. The overall relationship between diagnosis and titre is, however, highlighted by examining the percentage of patients in each diagnostic category as maximum ANCA titre increased (figure 3).

Predictive value theory ${ }^{17}$ allows the positive predictive value (PPV) of ANCA at each titre to be calculated. The PPVs have been calculated both for the total patient group ( $n=301$, table 4) and also excluding those in whom there was no final diagnosis or the diagnosis was acute/ chronic renal failure of unknown aetiology $(n=244$, table 5$)$. The effect of co-existent ANA has also been determined (table 4). In this context, the PPV is the percentage of all positive ANCA results which correctly indicated that the diagnosis was one of those defined in Group 1.

\section{Anti-myeloperoxidase testing}

In addition to indirect immunofluorescence, all positive sera were tested using an ELISA to detect antibodies with anti-myeloperoxidase (anti-MPO) specificity. Only 42 patients had positive anti-MPO antibodies ( $>20 \mathrm{EU}), 26$ of whom had a diagnosis in the vasculitic category; the remaining 16 patients had rheumatoid arthritis (4), systemic lupus erythematosus (1), undifferentiated connective tissue disease (1) and a non-vasculitic diagnosis (10). Thirteen of the anti-MPO patients $(31 \%)$ had cANCA pattern by immunofluorescence, seven of whom had vasculitic disorders.

The PPV of a positive anti-MPO result $(>20 \mathrm{EU})$ is calculated as $62 \%$; For the combination of a positive pANCA by indirect immunofluorescence and anti-MPO by ELISA $(>20 \mathrm{EU})$ the PPV was $66 \%$; this figure was not significantly affected by the presence or absence of an ANA in the serum (PPV $=65 \%$ when ANA also present (17/29), PPV $=67 \%$ when ANA not detected (12/29)). Increasing the ELISA cut-off level to 50 EU increased the PPV to $81 \%(n=21)$ but this showed no further improvement when the presence or absence of ANA was considered.

A total of $154(84 \%)$ pANCA-positive patients were negative by anti-MPO ELISA, despite the fact that 45 of them had relatively high maximum pANCA titres of 1:160 (22), $1: 320$ (13), $1: 640$ (7), $1: 1280(2)$ and $1: 2560(1)$. The remaining $109(70.8 \%)$ had pANCA values which did not exceed a titre of $1: 80$. The PPV of this pANCA positive, anti-MPO negative group was only $11 \%$ overall $(n=154)$; if there were co-existent ANA the PPV was reduced to $5 \%(n=86)$. The ANA negative subgroup had a PPV of $19 \%(n=67)$. 


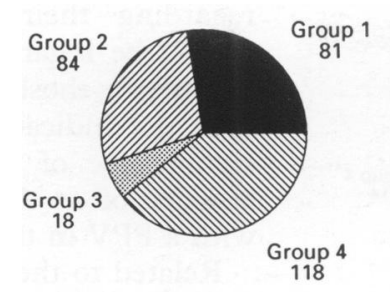

A

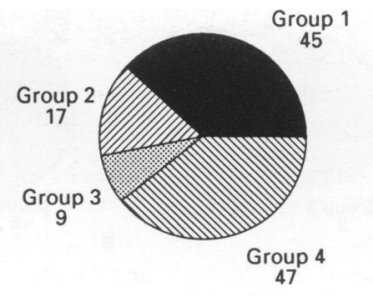

B

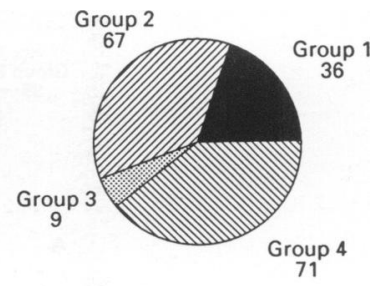

Figure 3 Patient numbers in each diagnostic group with subdivision into cANCA and pANCA positive groups. (A) Disease classification $(n=301)$; (B) cANCA $(n=118)$; (C) pANCA $(n=183)$

Table 4 Positive predictive values (PPV percentages) of ANCA at increasing titre and in relation to ANA

\begin{tabular}{|c|c|c|c|c|c|c|}
\hline \multirow[b]{2}{*}{ Titre } & \multicolumn{3}{|c|}{$p A N C A$} & \multicolumn{3}{|c|}{$c A N C A$} \\
\hline & Total & $\begin{array}{l}A N A \\
+v e\end{array}$ & $\begin{array}{l}A N A \\
-v e\end{array}$ & Total & $\begin{array}{l}A N A \\
+v e\end{array}$ & $\begin{array}{c}A N A \\
-v e\end{array}$ \\
\hline All & 20 & 15 & 27 & 38 & 32 & 40 \\
\hline $\begin{array}{l}\geqslant 1: 20 \\
\geqslant 1: 80 \\
\geqslant 1: 160 \\
\geqslant 1: 320 \\
\geqslant 1: 640\end{array}$ & $\begin{array}{l}22 \\
28 \\
32 \\
39 \\
53\end{array}$ & $\begin{array}{l}15 \\
20 \\
22 \\
31 \\
50\end{array}$ & $\begin{array}{l}33 \\
45 \\
45 \\
60 \\
57\end{array}$ & $\begin{array}{l}46 \\
55 \\
60 \\
67 \\
75\end{array}$ & $\begin{array}{l}32 \\
31 \\
25 \\
29 \\
-\end{array}$ & $\begin{array}{l}50 \\
61 \\
70 \\
80 \\
90\end{array}$ \\
\hline
\end{tabular}

Table 5 Positive predictive values (PPV percentages) of ANCA at increasing titre excluding patients from Group 4 with no final diagnosis or a diagnosis of acute/chronic renal failure of unknown aetiology

\begin{tabular}{lll}
\hline Titre & $p A N C A$ & $c A N C A$ \\
\hline All & 24 & 46 \\
$\geqslant 1: 20$ & 26 & 53 \\
$\geqslant 1: 80$ & 33 & 59 \\
$\geqslant 1: 160$ & 38 & 67 \\
$\geqslant 1: 320$ & 42 & 78 \\
$\geqslant 1: 640$ & 60 & 82 \\
\hline
\end{tabular}

\section{Antinuclear (ANA) and other autoantibodies}

ANA was detected in $125 / 301$ patients, 30 $(24 \%)$ had detectable antibodies to dsDNA, $103(82.4 \%)$ were pANCA, and $22(17.6 \%)$ cANCA. Of the 103 pANCA and ANA positive sera, 17 had positive anti-MPO by ELISA and of the remaining 86, 59 of the ANA were of the same isotype as the ANCA (IgG). In 51 of these samples the titres of ANA and ANCA were either unequal or divergent in repeated samples. The eight patients whose ANA and ANCA titres were equal had diagnoses of lupus (3), rheumatoid arthritis (2), chronic active hepatitis (2) and one with no diagnosis. Whilst there is some potential for difficulty in interpretation of indirect immunofluorescence when ANCA and ANA co-exist, by utilising the above strategies, we were able to reduce the extent of the problem. The frequency of other autoantibody detection is summarised in table 6.
Table 6 Other autoantibodies detected

\begin{tabular}{lr}
\hline Autoantibody & No patients $(n=301)$ \\
\hline Antinuclear & 125 \\
Rheumatoid factor & 51 \\
Anti-dsDNA & 31 \\
Anti-smooth muscle & 37 \\
Anti-parietal cell & 28 \\
Anti-mitochrondrial & 2 \\
Anti-centromere & 2
\end{tabular}

\section{Discussion}

ANCA detection has an important role in the early identification and monitoring of vasculitic disorders. ${ }^{2,3}$ We have carefully and critically reviewed the medical records of $92 \%$ of all patients in whom ANCA were detected in the Northern Irish population during a 45-month period and our study data have supported this assertion in so far as $81(27 \%)$ of our patients had one of the vasculitic conditions defined in Group 1. However, we have found that ANCA occur more frequently in patients with nonvasculitic conditions. In each case the decision to test for ANCA was clinician-led and not part of a laboratory policy or profile and our results therefore reflect actual clinical practice. We have defined the relative frequency of the non-vasculitic conditions amongst ANCApositive patients and this has improved our understanding of the appropriate place of ANCA in clinical practice. In audit terms this work is a 'partial audit, ${ }^{23}$ as although we have observed practice and as a result can make certain recommendations, we have not yet 'closed the loop' by observing the effect of these changes.

ANCA were detected in patients throughout the adult age range, and there was a slight female predominance (1.23:1) amongst our patients. There was no evidence of geographical clustering of patients nor of a significant association with cigarette smoking.

The finding that only 81 of our patients had a diagnosis from Group 1 appears at odds with previous reports of high sensitivity and specificity of ANCA for vasculitic conditions. Sensitivity and specificity are highly dependent on the degree of pre-selection of the study group and it was not our aim to calculate sensitivity and specificity in this study. We 

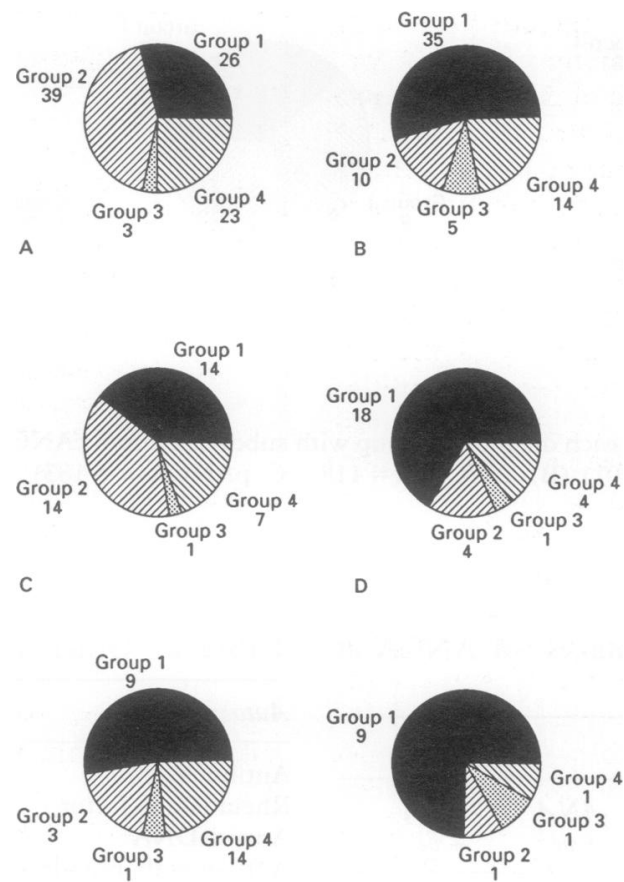

Figure 4 Increasing percentage of patients with vasculitic diagnosis as titre of ANCA increases. (A) pANCA $\geqslant 1: 80(n=91)$; (B) cANCA $\geqslant 1: 80(n=64)$; (C) pANCA $\geqslant 1: 320(n=36) ;(\mathrm{D})$ cANCA $\geqslant 1: 320$ $(n=27) ; \quad(\mathrm{E}) \quad$ pANCA $\geqslant 1: 640 \quad(n=17) ; \quad(\mathrm{F})$ cANCA $\geqslant 1: 640(n=12)$

were, however, concerned to establish the PPV of ANCA in routine clinical practice. PPV tells the clinician how certain he may be that a positive ANCA result means that his patient has a vasculitic condition. The overall PPV was $20 \%$ for all pANCA and $38 \%$ for all cANCA. These figures are comparable to those reported by Davenport et $a l^{4}$ who concluded therefore that " . . a diagnosis of necrotising vasculitis could neither be made nor refuted on the basis of an ANCA test alone ..." ". Whilst in general agreeing with this view, we still see diagnostic value in specific circumstances. Our recognition that non-vasculitic diagnoses tended to be associated with lower titre ANCA than the vasculitic group enabled us to stratify patients according to the maximum titre of ANCA detected (figure 2) and demonstrate increasing PPV for vasculitis with increasing titres (tables 4 and 5). cANCA had greater PPV at all titres than pANCA, however it was only with cANCA at a titre of greater/equal to 1:640 that we saw a PPV at a level $(75 \%)$ which could make one reasonably confident of diagnosis in the appropriate clinical setting. Importantly we found that the co-existence in serum of an ANA had the effect of reducing the PPV for vasculitis, irrespective of the titre of ANCA detected. One potential explanation of this phenomenon could be the misinterpretation of an ANA as a pANCA when seen on the neutrophil substrate. This is unlikely to be the case, however, as a similar effect was seen when cANCA was detected. It is highly unlikely that an ANA could cause a 'false-positive' cANCAstaining pattern. Rather, the detection of ANA in these patients appears to be informative regarding their underlying diagnoses and should be regarded as a helpful indicator in identifying those patients in whom the ANCA does not indicate vasculitis. In contrast, the significance of a high-titre cANCA in the absence of an ANA is clearly much greater, with a PPV in this study of $90 \%$.

Related to the issue of clinical selection for testing, we found that positive ANCA results in the patients of renal physicians were associated $c$ with a vasculitic diagnosis in $61 \%$ of cases whereas the corresponding figure from general physicians was $29 \%$. This difference may be related to many factors including patterns of referral and biopsy policies but it suggests that the selection of patients for ANCA testing is important.

We found that $220 / 301(73 \%)$ of all our $\vec{\circ}$ ANCA-positive patients had diagnoses outside Group 1 . This was clearly more likely with the detection of pANCA (147 (87\%)) than with $\overline{8}$ cANCA $(73(62 \%))$ and this lack of specificity 3 of pANCA has previously been recognised. ${ }^{6}$

The connective tissue disorders accounted for $28 \%$ of the ANCA detected, with $f$ rheumatoid arthritis comprising 55/301 $(18.2 \%)$ of the ANCA-positive patients. of Previous reports suggested that ANCA did not $\frac{\text { I }}{2}$ occur in uncomplicated rheumatoid arthritis $\vec{O}$ but only in the subset with Felty's syndrome. ${ }^{18}$ We have previously found that non-Felty's arthritis patients were frequently ANCA positive and that these patients tend to be the $\vec{\theta}$ rheumatoid-factor-positive subgroup with a of high frequency of extra-articular involvement and a requirement for immunosuppressive therapy. ${ }^{19}$ Our results confirm that view as have larger studies confined to rheumatoid arthritis patients. $^{20}$ Whether or not this association implies a prognostic value for ANCA testing in rheumatoid arthritis remains open to question. Of the 13 patients with ulcerative colitis or Crohn's disease, eight had cANCA. Recent work ${ }^{9,10}$ has shown that ANCA in inflammatory bowel disease is either pANCA or the atypical $\mathrm{XANCA}$ and that the antigenic specificity is not myeloperoxidase. Our study does not describe this atypical staining pattern as it predates its acceptance as a distinct pattern. Consequently the more accurate definition of indirect immunofluorescence now available would help $\frac{D}{2}$ to distinguish them from the vasculitic group, with the likelihood of increasing slightly the of PPV of both $p$ and cANCA.

The subgroup of patients with non- N vasculitic/no diagnosis were found to have $\omega$ lower ANCA titres than those patients with vasculitic diagnoses; this proved helpful in defining increasing PPV with increasing ANCA titre. The diagnoses found in this group 0 were very heterogeneous (see table 2). Of the 23 patients with renal failure (acute/chronic), 13 were seen by specialist renal physicians who found no other evidence for vasculitic renal $\bar{r}$ disease. The group with undefined acute/ 8 chronic renal failure along with those in whom no final diagnosis was achieved, clearly could include some patients with unrecognised vasculitic illness and potentially could have falsely lowered our PPVs. PPVs were therefore cal- 
culated with these patients excluded (table 4), however no significant change in PPV was identified. The occurrence of ANCA in infection has previously been a source of debate..$^{6,7,21}$ Six cases had cANCA (three respiratory infections and three urinary tract infections) and four pANCA (two respiratory, one paracolic abscess and one infective endocarditis). In addition to our previous report of four ANCApositive patients with malignancy of the respiratory or urinary tracts and renal vasculitis, ${ }^{8}$ we now add a further 13 ANCA-positive patients with malignant disease in whom there was no evidence of vasculitis.

Interpretation of the ANCA indirect immunofluorescence findings requires observer experience and consistency, and it remains the 'gold standard' in clinical practice. There is, however, a degree of subjectivity which makes the development of solid-phase, quantitative assays desirable. Our knowledge of the multiple target antigens of ANCA demonstrates that several suitably standardised assays would be required to replace indirect immunofluorescence. At the time of the study only the anti-MPO ELISA was available to us but this provided useful additional data. Antibodies to MPO were detected in only a subset of those patients with ANCA detected by indirect immunofluorescence and although they had a better PPV $(62 \%)$, they also lacked specificity, occurring in patients from all four groups. The improvement of PPV from $20 \%$ to $66 \%$ when a positive result is obtained in both indirect immunofluorescence and anti-MPO ELISA (irrespective of immunofluorescent titre) makes a strong case for the inclusion of such an assay in routine practice. We have previously reported that in a small number of patients the anti-MPO assay provided a positive result earlier in the course of illness than did immunofluorescence. ${ }^{25}$

In conclusion, therefore, we find that ANCA occur more frequently in non-vasculitic conditions when the population is otherwise unselected. Whilst the subgroup with rheumatoid arthritis showed certain clinical characteristics, it would be over simplistic to suggest that ANCA detected in non-vasculitic disease are related to specific patterns of organ involvement or indeed disease severity. If ANCA are to remain useful tools in the diagnosis and

1 Davies DJ, Moran JE, Niall JF, Ryan GB. Segmenta necrotising glomerulonephritis with antineutrophil

2 van der Woude FJ, Rasmussen N, Lobatto S, et al. Autoantibodies against neutrophils and monocytes: tool for diagnosis and marker of disease activity in Wegener's granulomatosis. Lancet 1985; 1: 425-9.

3 Tervaert JWC, Huitema MG, Hene RJ, et al. Prevention of relapses in Wegeners' granulomatosis by treatment based on antineutrophil cytoplasmic antibody titre. Lancet 1990; 336 709-11.

4 Jennette JC, Falk RJ. Antineutrophil cytoplasmic autoantibodies and associated diseases: a review. $A m \mathcal{F}$ Kidney Dis 1990; 15: 517-29.

5 Gross WL, Schmitt WH, Csernok E. ANCA and associated diseases: immunodiagnostic and pathogenetic aspects. Clin Exp Immunol 1993; 91: 1-12.

6 Gross WL, Hauschild S, Mistry N. The clinical relevance of ANCA in vasculitis. Clin Exp Immunol 1993; 93 (suppl 1): ANCA.

7 Efthimou J, Spickett G, Lane D, Thompson A. AntineutEfthimou J, Spickett G, Lane D, Thompson A. Antineut-
rophil cytoplasmic antibodies, cystic fibrosis, and infection (letter) Lancet 1991; 337: 1037-8.
Learning/summary points

- ANCA may be detected in the sera of patients with a wide variety of clinical conditions

- clinical interpretation of ANCA results must include the possibility of the wide range of non-vasculitic disorders described

- the predictive value of ANCA for a vasculitic disorder is dependent on the titre of ANCA, pattern cANCA/pANCA, and the presence or absence of associated ANA

- the predictive value of pANCA is improved when further characterised as myeloperoxidase specific

- high-titre cANCA remains highly predictive for an underlying vasculitic disorder

Box 2

monitoring of patients with primary vasculitides we must be aware of the relative frequencies of other diseases which can result in a positive ANCA. The investigation of patients for vasculitic conditions should include ANCA testing as part of a rational investigative scheme along with other serology, appropriate radiology, biochemistry and histological examination of biopsy tissue. As a result of this study we have increased our screening dilution to $1: 20$ to reduce the detection of very low titre ANCA and we continue to use the anti-MPO system to improve the PPV of pANCA. The coexistence of an ANA indicates that the likely diagnosis is not one of those we have defined as Group 1; however, the combination of a high-titre cANCA in the absence of an ANA is highly predictive for vasculitis. The combination of positive pANCA by indirect immunofluorescence and anti-MPO by ELISA significantly improves the PPV. ANCA testing is not suitable as a screening test for vasculitis and positive ANCA results, in patients without good clinical evidence for vasculitis, should be interpreted with care.

We would like to express our thanks to our clinical colleagues throughout Northern Ireland without whose help this study would not have been possible. In particular our thanks are due to the physicians of the Regional Nephrology Unit, Belfast City Hospital and the Department of Rheumatology, Musgrave Park Hospital, Belfast. Dr IN Bruce is in receipt of a Royal Victoria Hospital, Clinical Research Fellowship.

8 Edgar JDM, Rooney DP, McNamee P, McNeill TA. An association between ANCA-positive renal disease and malignancy. Clin Nephrol 1993; 40: 22-5.

9 Saxon A, Shanahan F, Landers C, et al. A distinct subset of antineutrophil cytoplasmic antibodies is associated with inflammatory bowel disease. $\mathcal{F}$ Allergy Clin Immunol 1990 ; 86: $202-10$.

10 Rump JA, Scholmerich J, Gross V, et al. A new type of perinuclear anti-neutrophil cytoplasmic antibody (pANCA) in active ulcerative colitis but not in Crohn's disease. Immunobiology 1990; 181: 406-13.

11 Coremans IEM, Hagen EC, Daha MR, van der Woude FJ, van der Voort, Breedfeld FC. Autoantibodies to neutrophil cytoplasmic enzymes in Felty's syndrome. Clin Exp Immunol 1993; 93: 255-62.

12 Wiik A. Delineation of a standard procedure for indirect immunofluorescent detection of ANCA. Acta Pathol Microbiol Immunol Scand 1989; 97 (suppl): 12-3.

13 Falk RJ, Terrell RS, Charles LA, Jennette JC. Antineutrophil cytoplasmic autoantibodies induce neutrophils to degranulate and produce oxygen radicals in vitro. Proc Natl Acad Sci USA 1990; 87: 4115-9. 
14 Ewart BH, Jennette JC, Falk RJ. The pathogenic role of antineutrophil cytoplasmic autoantibodies. Am $\mathcal{F}$ Kidney Dis antineutrophil cyto

15 Gross WL, Csernok E, Schmitt WH. Antineutrophil cytoplasmic autoantibodies: immunobiological aspects. Klin Wochenschr 1991; 69: 558-66.

16 Cupps TR. Cardiac and vascular diseases. In: Stites DP, Terr AI, eds. Basic and clinical immunology 7th edn. Connecticut: Appleton and Lange, 1991; pp 493-505.

17 Gallen RS. Use of predictive value theory in clinical immunology. In: Rose NR, Friedman H, Fahey JL, eds. Manual of clinical laboratory immunology 3 rd edn. Washington: American Society of Microbiology, 1986; pp 966-71.

18 Juby C, Johnston C, Davis P, Russell AS. Antinuclear and antineutrophil cytoplasmic antibodies (ANCA) in the sera of patients with Felty's syndrome. Br $\mathcal{F}$ Rheumatol 1992; 31: 185-8.

19 Edgar JDM, Conlan SK, Bell AL, McMillan SA. Antineutrophil cytoplasmic antibodies in rheumatoid arthritis. (letter) $B r \mathcal{F}$ Rheumatol 1992; 31: 859-60.

20 Braun MG, Csernok E, Schmitt W, Hauschild S, Gross WL. Anti-neutrophil cytoplasmic antibodies (ANCA) and extraarticular involvement in rheumatoid arthritis (RA). (abstract) Clin Exp Immunol 1993; 93 (suppl 1): 33.
21 Schmitt WH, Csernok E, Gross WL. ANCA and infection. (letter) Lancet 1991; 337: 1416-7.

22 Ramirez G, Khamashta MA, Hughes GRV. The ANCA test: its clinical relevance. Ann Rheum Dis 1990; 49: 741-2.

23 Lawrence M, Griew K, Derry J, Anderson J, Humphreys J. Auditing audits: use and development of the Oxfordshire Medical Advisory Group rating system. BMF 1994; 309: 513-6.

24 Davenport A, Lock RJ, Wallington TB, Feest TG. Clinical significance of anti-neutrophil cytoplasm antibodies detected by a standardised indirect immunofluorescence assay. Qf Med 1994; 87: 291-9.

25 Edgar JDM, Cooper M, Conlan SK, McMillan SA, McNeill TA. Use of an ELISA system to detect antimyeloperoxidase autoantibodies in systemic vasculitis. (abstract) Clin Exp Allergy 1992; 22: 135. 